\title{
Journalists and Twitter: How Indian News Organization Adapt to a Social Networking
}

\author{
*Dr. Devadas Mb \\ Assistant Professor Dept. Of Media and Communication \\ Central University of Tamil Nadu Thiruvarur-610004
}

\begin{abstract}
[Abstract: This paper examines new Para-journalism forms such as micro-blogging as "awareness systems" that provide journalists with more complex ways of understanding and reporting on the public communication. Traditional journalism defines fact as information and quotes from official sources, which have been identified as forming the vast majority of news and information content. This model of news is in flux, however, as new social media technologies such as Twitter facilitate the instant, online dissemination of short fragments of information from a variety of official and unofficial sources. This paper draws from literature on new communications technologies in computer science to suggest that these broad, asynchronous, lightweight and always-on systems are enabling citizens to maintain a mental model of news and events around them, giving rise to awareness systems this paper describes as ambient journalism. The emergence of ambient journalism brought about by the use of these new digital delivery systems and evolving communications protocols raises significant research questions for journalism scholars and professionals. This research offers an initial exploration of the impact of awareness systems on journalism norms and practices. It suggests that one of the future directions for journalism may be to develop approaches and systems that help the public negotiate and regulate the flow of awareness information, facilitating the collection and transmission of news.]
\end{abstract}

Key Words: Awareness systems; Internet; Journalism; Micro-blogging; Social Media; Twitter

\section{Introduction}

Twitter is one of a range of new social media technologies that allows for the online and instant dissemination of short fragments of data from a variety of official and unofficial sources. The micro-blogging service has emerged as a platform to help organize and disseminate information during major events like the 2002 Gujarat Godra incident, the 2009 Lokasabha elections in India, the Mumbai massacre and the Anna Hazare's are protests against corruption of 2013.

The emergence of Twitter as a significant form of communication was reflected in the request by the Indian Central government asking Twitter to delay routine maintenance during the Lokpal bill in Parliment as the service emerged as an important tool used by people inside India to coordinate protests against the Central government. Media restrictions led websites of The Times of India, The Indian express newspaper in Bangalore and others to publish mix of unverified accounts from Twitter, YouTube and blogs as "amateur videos and eyewitness accounts became the de facto source for information".

The micro-blogging service illustrates what Hayek described years before the invention of the Internet as "the knowledge of particular circumstances of time and place". The development of a system that has created new modes of organizing knowledge that rely on large, loosely organized groups of people working together electronically. A variety of terms have been used to describe this: crowd-sourcing, wisdom of crowds, peer production, suggests that the phrase "collective intelligence" is the most useful to describe this phenomenon, which they broadly define as groups of individuals doing things collectively that seem intelligent. I suggest that micro-blogging systems that enable millions of people to instantly to communicate, share and discuss events happening around them are one of these new forms of collective intelligence.

This paper examines micro-blogging as one of a range of new media technologies that enable citizens to "obtain immediate access to information held by all or at least most, and in which each person can instantly add to that knowledge". It argues that new Para-journalism forms such as micro-blogging are "awareness systems,' providing journalists with more complex ways of understanding and reporting on the subtleties of public communication.

Traditional journalism defines fact as information and quotes from official sources, which in turn has been identified as forming the vast majority of news and information content. This news model is in a period of transition; however, as new social media technologies like Twitter now facilitate the immediate dissemination of digital fragments of news and information from official and unofficial sources over a variety of systems and devices. This paper draws from literature on new communications technologies in computer science to suggest 
that these broad, asynchronous, lightweight and always-on communication systems are creating new kinds of interactions around the news, and are enabling citizens to maintain a mental model of news and events around them, giving rise to what this paper describes as ambient journalism.

\section{Definition of micro-blogging}

Micro-blogging has been defined as a new media technology that enables and extends our ability communicate, sharing some similarities with broadcast. It allows "users to share brief blasts of information to friends and followers from multiple sources including websites, third-party applications, or mobile devices". Several services including Twitter, Jaiku and Tumbler provide tools that enable this form of communication, although status updates embedded within websites such as Face book, MySpace, and LinkedIn offer similar functionality.

One of the most popular micro-blogging platforms is Twitter. Between Jan 2008 and December 2013, the number of Twitter accounts rose from 1 million to 52 million. This growth was partially fuelled by increased media attention to Twitter and a higher profile as celebrities such as ex cricketer Sachin Tendulkar and Gujarat Chief Minister Narendra Modi adopted the service. Despite the rapid uptake, Twitter is still only used by a select number of people. In the India, only $10 \%$ of Indian adults use Twitter or similar tools and research suggests that $8 \%$ of prolific Twitter users account for over $70 \%$ of messages.

But there are indications that Twitter users tend to be the people who are interested and engaged with the news. Studies show that the largest single group of tweeters, making up 32 per cent of the total, are between the ages of 35 to 49 , and that the average Twitter user is two to three times more likely to visit a leading news website than the average person.

A study of micro-blogging must consider the communication activities and practices that have developed around it. Twitter is a highly flexible system that routes messages sent from a variety of devices to people who have chosen to receive them in the medium they prefer. It asks users the question: "What are you doing?" Messages are limited to 140 characters as the system was originally designed for messages to be shared via SMS, but there are no limits on how many updates a user can send. The "tweets" can be shared publicly or within a social network of followers. As with many new technologies, users have extended their use of Twitter to more than just answering the initial question. The service has been described as an example of end-user innovation (Johnson, 2009) as users have embraced the technology and its affordances to develop conventions such as the use of hash tags and the @ reply for uses beyond the original intentions of its creators.

Micro-blogging is a relatively nascent phenomenon and as such is under-developed as an area of study, especially in journalism research. Studies such one as by Java et al (2007) have looked at the motivation of users, concluding that micro-blogging fulfils a need for a fast mode of communication that "lowers users' requirement of time and thought investment for content generation" (Java et al., 2007 p2). In their analysis on user intentions, they found that people use Twitter for four reasons: daily chatter, conversation, sharing information and reporting news. At least two of these - sharing information and reporting news - can be considered as relevant to journalism, though arguably so could daily chatter and conversation around current events. Additionally two of the three main categories of users on Twitter defined by Java et al (2007) information source and information seeker - are also directly relevant to journalism.

\section{Twitter and journalism}

There has been a rapid uptake of Twitter by media professionals, with many news outlets considering Twitter an essential addition to a reporter's toolkit (Posetti, 2009). Sites such as MuckRack.com also aggregate journalists' tweets. Twitter has been quickly adopted in newsrooms as a mechanism to distribute breaking news quickly and concisely or as a tool to solicit story ideas, sources and facts. In the Indian, national newspapers had 250 official Twitter accounts by May 2014, with a total of just over one million followers. In a sign of how far Twitter has come, the India-based Times Now News Channel appointed a Twitter correspondent in March 2012 who would be "scouring Twitter for stories and feeding back, giving Times Now News a presence in the Twitter sphere.

The relative newness of micro-blogging means there is limited academic literature on the impact of the phenomenon on journalism. When the topic is broached in the mainstream media, it is framed within the context of established journalism norms and values. There has been a degree of bewilderment, skepticism and even derision towards Twitter from seasoned journalists. Setting aside ridicule, there has been discussion on whether the breadth and depth of news reporting would suffer as more reporters sign up to Twitter.

Particular concern has been how journalists should adopt social media within existing ethical norms and values (Posetti, 2009), leading news organizations such as Times of India, The Hindu and Deccan Herald to institute social media policies specific to Twitter to bring its use in line with established professional practices.

Micro-blogging has been discussed in the context of citizen journalism, where individuals perform some of the institutionalized communication functions of the professional journalist, often providing the first 
accounts, images or video of a news event. The value of this user-generated content is assessed by the professional norms and values that are presumed to guarantee the quality of the information. The issue commonly discussed in media commentaries on the role of Twitter in journalism is the veracity and validity of messages.

Concerns by journalists that many of the messages on Twitter amount to unsubstantiated rumors and wild inaccuracies are rose when there is a major breaking news event, from the Mumbai bombings, Anna Hazare against corruption protests and 2014 Loksabha election in India. The unverified nature of the information on Twitter has led journalists to comment that "it's like searching for medical advice in an online world of quacks and cures" (Goodman, 2009) and "Twitter? I won't touch it. It's all garbage" (Stelter, 2009). The professional and cultural attitudes surrounding Twitter have their roots in the working routines and entrenched traditional values of a journalistic culture which defines the role of the journalist as providing a critical account of daily events, gathered, selected, edited and disseminated by a professional organization. If reflects the unease in adopting a platform which appears to be at odds with journalism as a "professional discipline for verifying information". While more research is needed in this area, there are indications that journalism norms are bending as professional practices adapt to social media tools such as micro blogging.

News organizations published "minute-by-minute blogs with a mix of unverified videos, anonymous Twitter messages and traditional accounts from Indian. Six months earlier, the Times Now and CNN-IBN included unverified tweets filtered by journalists alongside material from correspondents in its breaking news coverage of the Mumbai bombings, 2014 Loksabha Election in India. The CNN-IBN and Times Now justified its decision on the grounds that: On a major unfolding story there is a case also for simply monitoring, selecting and passing on the information we are getting as quickly as we can, on the basis that many people will want to know what we know and what we are still finding out, as soon as we can tell them (Herrmann, 2009). This approach still requires journalists to an interpretive standpoint as to utility of a tweet around a news event or topic, making a choice as to what to exclude or include, so that the professional remain the guardians of what content is published. By filtering and selecting what tweets to publish, the gatekeeper role is maintained and enforced. In this context, journalists tend to apply existing news values to determine if a specific tweet is newsworthy, dismissing content that might be considered as "snarky and trivia" (Farhi,2009). Social media technologies like Twitter are part of a range of Internet technologies enabling the disintermediation of news and undermining gate keeping function of journalists.

Micro-blogging can be seen as a form of participatory or citizen journalism, where citizens report without recourse to institutional journalism (Thurman and Hermida, 2009). It forms part of a trend in journalism that Deuze has described as a shift from "individualistic, 'top-down' mono-media journalism to team-based, 'participatory' multimedia journalism". Indeed, as Hall argues, "the roles that journalism assigned to itself in the mid-nineteenth century as gatekeeper, agenda-setter and news filter, are all placed at risk when its primary sources become readily available to its audiences". However, this paper contends that while micro-blogging services such as Twitter can be situated within the trend in citizen journalism, it should also be considered a system of communication with its own media logic, shapes and structures. While Twitter can be used to crowd source the news, where a large group of users come together to report on a news event, this paper argues that the institutionally structured features of micro-blogging are creating new forms of journalism, representing one of the ways in which the Internet is influencing journalism practices and, furthermore, changing how journalism itself is defined.

Micro-blogging presents a multi-faceted and fragmented news experience, marking a shift away from the classical paradigm of journalism as a framework to provide report and analyses of events through narratives, producing an accurate and objective rendering of reality. Indeed, services like Twitter are a challenge to a news culture based on individual expert systems and group think over team work and knowledge sharing suggest, "to unlock the potential of collective intelligence, managers instead need a deeper understanding of how these systems work." This paper seeks to contribute an understanding of Twitter by introducing the concept of ambient journalism. I see new media forms of micro-blogging as "awareness systems," providing journalists with more complex ways of understanding and reporting on the subtleties of public communication. Established journalism is based on a content-oriented communication, whereas Twitter adds an additional layer which can be considered as what has been referred to as connectedness-oriented communication. In an awareness system, value is defined less by each individual fragment of information that may be insignificant on its own or of limited validity, but rather by the combined effect of the communication. The next section defines awareness systems in more detail, with an eye towards understanding its application to journalism.

\section{Micro-blogging as ambient journalism}

Drawing on the literature in the field of human-computer interaction, this paper suggests that broad, asynchronous, lightweight and always-on communication systems such as Twitter are enabling citizens to maintain a mental model of news and events around them. In this context, Twitter can be considered as an 
awareness system. Awareness systems are computer-mediated communication systems "intended to help people construct and maintain awareness of each others' activities, context or status, even when the participants are not co-located". Awareness systems have largely been discussed in the context of Computer- Supported Cooperative Work, with a focus on the notion of connecting remote co-workers by audio/video links. But there have also been critiques of the benefits of awareness and even criticism of the term awareness as vague and problematic, often used in contradictory ways in the literature. However, the emergence of the web, coupled with increasingly affordable and ubiquitous information communication technologies have helped foster a renewed research interest in awareness systems. One focus of research is awareness systems for use in personal settings, where lightweight, informal communication systems help people maintain awareness of each other. These systems are always-on and move from the background to the foreground as and when a user feels the need to communicate. Scholars suggest that awareness systems represent the next step in the evolution of communication technologies that have increased the frequency and amount of information transfer.

Instant messaging and mobile phones provide awareness cues about others who are currently online and Internet-connected photo frames and robots permit users to display awareness information, either from broadcasts such as the weather or from members of one's social network. As this technology becomes more affordable, with greater quality and diversity, awareness systems offer tremendous potential for innovation, with a wide range of forms and contexts for transforming the space around us. This paper adopts the definition of awareness proposed by Chalmers as "the ongoing interpretation of representations i.e. of human activity and of artifacts". I suggest that this definition can be applied to social media networks such as Twitter, with messages considered as both the representations of human activity and as artifacts.

As such, Twitter becomes a system where news is reported, disseminated and shared online in short, fast and frequent messages. It creates an ambient media system that displays abstracted information in a space occupied by the user of the service. In this system, a user receives information in the periphery of their awareness. An individual tweet does not require the cognitive attention of, for example, an e-mail message. Indeed, the value does not lie in each individual fragment of news and information, but rather in the mental portrait created by a number of messages over a period of time. This is what I describe as ambient journalism an awareness system that offers diverse means to collect, communicate, share and display news and information, serving diverse purposes.

The system is always-on but also works on different levels of engagement in terms of awareness, creating an ecosystem where "a single user may have multiple intentions or may even serve different roles in different communities" (Java et al., 2007 p8). The issues for journalism professionals and researchers is how individuals assign meaning to information from others, how they selectively attend to this information and how intentions are assigned to the information (Markopoulos et al., 2009).

In the literature on ambient media, scholars talk about improving people's quality of life by creating the desired atmosphere and functionality through intelligent, personalized, interconnected digital systems and services, with intelligent devices embedded in everyday objects. In his discussion of ambient media,

Lugmayr (2006) argues that today's technology is too complex, dominated by an individual's struggle to command the technology to do what they want. Instead, he suggests, we should aim to create media systems that can know what an individual desires and act autonomously on their behalf. If we consider Twitter as a form of ambient journalism, then the issue becomes the development of systems that can identify, contextualize and communicate news and information from a continuous stream of 140- character messages to meet the needs of an individual. To understand this further, it is appropriate to consider what Weiser and Brown (1996) suggest are designs that provide varying levels of engagement by moving in and out of an individual's center of attention. They proposed the concept of calm technology in the response to the rise of ubiquitous computing, looking ahead towards a world where technology is embedded in the fabric of everyday life. Weiser and Brown talk about the need for systems that allow for information to attract attention at different levels of awareness, be it at the centre or periphery of our attention. With Twitter, such an approach would enable users to be aware of the ambient information in the periphery, but would also bring from the periphery of our attention into the centre of our attention as required.

\section{Suggested approaches in ambient journalism}

As an initial exploration into the impact of awareness systems on journalism norms and practices, the next section of this paper examines the implications of Twitter as ambient journalism. This paper has already discussed how the first reports of a news event are now coming from people at the scene in the form of a 140character message.

But as an awareness system, Twitter goes beyond being just a network for the rapid dissemination of breaking news from individuals at the location of an event. Rather, it can be seen as a system that alerts journalists to trends or issues hovering under the news radar. Twitter as a collective intelligence system that provides early warnings about trends, people and news. The immediacy and velocity of these micro-bursts of 
data, as well as potentially the high signal to noise ratio, presents challenges for the established practice of relying on the journalist as the filter for this information. During the Indian general election 2009, the volume of tweets mentioning Indian peaked at 321,774 in one hour, from a flow of between 12,000 and 1,00000 an hour. The need to reduce, select and filter increases as the volume of information grows, suggesting a need for information systems to aid in the representation, selection and interpretation of shared information The growing volume of content on micro-blogging networks suggests that one of the future directions for journalism may be to develop approaches and systems that help the public negotiate and regulate this flow of awareness information, facilitating the collection and transmission of news. This would enable journalists to, as Hayek (1979) urged, utilize the knowledge that is dispersed among individuals. The purpose of these systems would be to identify the collective sum of knowledge contain in the micro-fragments in a manner that would bring meaning to the data.

Bradshaw (2008) discusses some of the systems used to aggregate tweets at the time of the Japanese earthquake in 2011. Research in this area is in its preliminary phases with web applications such as Twitcoop, http://www.twitscoop.com/), that aim to detect trends in real-time and visualizes the "buzz" on Twitter with custom graphs that display the activity around words and topics. Another similar web application is NowPublic.com's Scan (http://labs.nowpublic.com/) seeks to track the micro-blogosphere to "search the conversations on these sites based on keywords" and "find undiscovered news photos and videos by ordinary people and hidden links to breaking news". These systems still rely on a journalistic interpretative standpoint as to the utility or interest in a topic, based on choices on what to include and exclude, suggesting there is still a filtering mechanism at work, albeit on a systems design level.

Considering Twitter as an awareness system also represents a shift in the consumption of news and information. In such systems, completeness of awareness is not the goal, as it would be if an individual were actively pursuing an interest in a specific news event by following it in print, broadcast or online. Instead of overburdening an individual with an endless stream of tweets, Twitter as an always-on, asynchronous awareness system informs but does not overburden you with information, and only makes you aware of them when you need them (Tugui, 2004). This notion draws on ideas advanced by Weiser and Brown of a future society dominated by calm technologies and the Internet, where technology will advance to the stage where it becomes embedded and invisible in people's lives. The extent to which such systems of ambient journalism allow citizens to maintain an awareness of the news events and the conversation around them would be a fertile area for future study.

The trend to share links on Twitter provides a mechanism for what Johnson describes as a customized newspaper, "compiled from all the articles being read that morning by your social network" (2009). In this context, tweets provide a diverse and eclectic mix of news and information, as well as an awareness of what others in your network are reading and what they consider important. The information transmitted is contentsoriented but also provides a context for the news-seeking activities of others on the network, which may make "visible the structure of implied communities," (Sarno, 2009).

There are concerns that "political partisans looking to enhance their own private echo chamber will be able to tune out opposing viewpoints more easily" (Johnson, 2009) but, as Sunstein (2006) argues, such a position may be too simplistic. This is an area that merits further exploration within the field of journalism studies, contributing to the discussion about whether Internet technologies are creating a "Daily Me" or a "Daily Us". Basing further research on an approach to networks such as Twitter as awareness systems, can, I suggest, help to contextualize the processes of the production, content, reception and circulation of news.

The link-based nature of many tweets, and the trend to re-send the links as a "retweet", can be analysed as both a form of data sharing and as a system for creating a shared conversation. This conversation can be considered as a form of ambient journalism. Since the retweets are not restricted by physical space, time or a delineated group, this creates what boyd et al (2009) argue is a distributed conversation that allows others to be aware of the content, without being actively part of it. In an initial exploration of the practice of retweeting, Boyd et al suggest that the "stream of messages provided by Twitter allows individuals to be peripherally aware of discussions without being contributors." This is significant in the context of engaging with audiences through the notion of journalism as a conversation (Gillmor, 2004). Scholars contend that awareness systems can be conceived as networks that engender information interactions and the development of a shared culture, which is particularly important for groups distributed across geography. Research is needed to determine how far Twitter, as an awareness system for news, is contributing to the creation or strengthening of social bonds. For example, the mass outpouring of tweets following the death of Michael Jackson in July 2009 has been described as an immediate and public "collective expression of loss". This is not to say that Twitter is replacing existing communication systems, but that Twitter, as an awareness system, is "enriching them, strengthening existing social bonds and enabling new kinds of interactions" (IJsselsteijn et al., 2003). 


\section{Conclusion}

As with most media technologies, there is a degree of hyperbole about the potential of Twitter, with proclamations that "every major channel of information will be Twitterfied in one way or another in the coming years" (Johnson, 2009). Furthermore, social media services are vulnerable to shifting and ever-changing social and cultural habits of audiences. While this paper has discussed micro-blogging in the context of Twitter, it is possible that a new service may replace it in the future. This paper contends that it is important to explore in more detail the qualities of micro-blogging - real-time, immediate communication, searching, link-sharing and the follower structure - and their impact on the way news and information is communicated. The emergence of ambient journalism brought about by the use of these new digital delivery systems and evolving communications protocols, in the case of this study Twitter, raises significant research questions for journalism scholars and professionals. This paper offers an initial exploration of the relationship between awareness systems and shifting journalism norms and practices. Twitter is, due to the speed and volume of tweets, a "noisy" environment, where messages arrive in the order received by the system. A future direction for journalism may be to develop approaches and systems that help the public negotiate and regulate the flow of awareness information, providing tools that take account of this new mode for the dissemination of news. Thus, journalists would be seen as sense makers, rather than just reporting the news. This broadens the journalist's role as proposed by Bardoel and Deuze of a professional "who serves as a node in a complex environment between technology and society, between news and analysis, between annotation and selection, between orientation and investigation" (2001 p101). In the case of ambient journalism, the role may be designing the tools that can analyze, interpret and contextualise a system of collection intelligence, rather than in the established practice of selection and editing of content through the prism of news values.

Micro-blogging, and Twitter specifically, are in the early stages of development. The significance of Twitter as a news and information platform will be largely influenced by its adoption, both in journalism and other spheres. The richness and utility of a place increases as people build up a past that involves it and a record of experiences. The challenge for researchers is to understand how this place becomes, in the words of Harrison and Dourish, "the understood reality" through a conversational and collaborative user experience. Examining Twitter as an awareness system, creating what I have called ambient journalism, provides a framework to analyze the emergent patterns of human behavior and data interaction that offer an understanding of this place. It shifts the journalistic discourse on micro-blogging away from a debate based on raw data to contextualized, significant information based on the networked nature of a synchronous, lightweight and always-on communication systems.

\section{References}

[1]. CASHMORE, PETE (2009) "Michael Jackson Dies: Twitter Tributes Now 30\% of Tweets", Mashable. http://mashable.com/2009/06/25/michael-jackson-twitter/, accessed June 25, 2009.

[2]. CHALMERS, MATTHEW (2002) “Awareness, Representation and Interpretation”, Computer Supported Cooperative Work, 11, 389-409.

[3]. CHENG, ALEX, EVANS, MARK AND SINGH, HARSHDEEP (2009) Inside Twitter: An In-Depth Look Inside the Twitter World: Sysomos.

[4]. COLES, MALCOLM (2009) "Newspapers on Twitter: How the Guardian, FT and Times are winning", http://www.malcolmcoles.co.uk/blog/newspapers-on-twitter/, accessed 6 July, 2009.

[5]. DAHLGREN, PETER (1996) "Media Logic in Cyberspace: Repositioning Journalism and its Publics", Javnost/The Public, 3, 5972.

[6]. DEUZE, MARK (2005) "What is Journalism? Professional Identity and Ideology of Journalists Reconsidered", Journalism, 6, 442464.

[7]. DEVOE, KRISTINA M. (2009) "Burst of Information: Microblogging”, The Reference Librarian, 50, $212-214$.

[8]. DUCATEL, K., BOGDANOWICZ, M., SCAPOLO, F., LEIJTEN, J. AND BURGELMAN, J-C. (2001).

[9]. GROSS, TOM, STARY, CHRIS \& TOTTER, ALEX (2005) "User-Centered Awareness in Computer- Supported Cooperative Work Systems: Structured Embedding of Findings from Social Sciences", International Journal of Human-Computer Interaction, $18,323-360$.

[10]. HALL, JIM (2000) Online Journalism: A Critical Primer, London: Pluto Press.

[11]. HARRISON, STEVE AND DOURISH, PAUL (1996) "Re-Place-ing Space: The Roles of Place and Space in Collaborative Systems", in CSCW '96: Proceedings of Conference on Computer Supported Cooperative Work, Boston: ACM Press, 67 -76.

[12]. HAYEK, FRIEDRICH A. VON (1979) Law, legislation and liberty : a new statement of the liberal principles of justice and political economy, Vol.3, The political order of a free people London: Routledge and Kegan Paul.

[13]. HEIL, BILL AND PISKORSKI, MIKOLJAI (2009) "New Twitter Research: Men Follow Men and Nobody Tweets", Harvard Business Publishing.

[14]. HERMIDA, ALFRED AND THURMAN, NEIL (2009) "A Clash of Cultures: The Integration of User-Generated Content within Professional Journalistic Frameworks at British Newspaper Websites”, Journalism Practice, 2, 343-356.

[15]. MARKOPOULOS, PANOS, IJSSELSTEIJN, WIJNAND, HUIJEN, CLAIRE, ROMIJIN, ONNO, AND PHILOPOULOS, ALEXANDROS (2003) "Supporting social presence through asynchronous awareness systems", in Riva, Giuseppe, Davide, Fabrizio, and Ijsselsteijn, Wijnand (eds.) Being There: Concepts, Effects and Measurements of User Presence in Synthetic Environments. Amsterdam: IOS Press, pp 261-278. 